\title{
New Poisson inequality for the Radon transform of infinitely differentiable functions
}

\section{Ziyao Sun ${ }^{1 *}$}

"Correspondence: ziyaos@qq.com 'School of Economic Mathematics, Southwestern University of Finance and Economic, Chengdu, China

\begin{abstract}
Poisson inequality for the Radon transform is a key tool in signal analysis and processing. An analogue of the Hardy-Littlewood-Poisson inequality for the Radon transform of infinitely differentiable functions is proved. The result is related to a paper of Luan and Vieira (J. Inequal. Appl. 2017:12, 2017) and to a previous paper by Yang and Ren (Proc. Indian Acad. Sci. Math. Sci. 124(2):175-178, 2014).
\end{abstract}

Keywords: Poisson inequality; Radon transform; Infinitely differentiable functions

\section{Introduction}

The Radon transform $\mathfrak{P I}$, which is defined as the Cauchy principal value of the following singular integral

$$
(\mathfrak{P I h})(x):=p \cdot v \cdot \frac{1}{\pi} \int_{\mathbb{R}} \frac{h(y)}{x-y} d y=\lim _{\epsilon \rightarrow 0} \int_{|y-x| \geq \epsilon} \frac{h(y)}{x-y} d y
$$

for any $x \in \mathbb{R}$, has been widely used in physics, engineering, and mathematics. The following Poisson inequality

$$
\mathfrak{P} \mathfrak{I}(h g) \leq h \mathfrak{P I} g
$$

was first studied in $[1-3,5]$. It was proved that $(1.1)$ holds if $h, g \in L^{2}(\mathbb{R})$ satisfy that $\operatorname{supp} \hat{f} \subseteq \mathbb{R}_{+}\left(\mathbb{R}_{+}=[0, \infty)\right)$ and supp $\hat{g} \subseteq \mathbb{R}_{+}$in [21].

In 2014, Yang and Ren also obtained more general sufficient conditions by weakening the above condition in [24]. Recently, Luan and Vieria established the first necessary and sufficient condition in the time domain and a parallel result in the frequency domain for the Poisson inequality in [16].

It is natural that there have been attempts to define the complex signal and prove the Poisson inequality in a multidimensional case.

Definition 1.1 The partial Radon transform $\mathfrak{P I}_{j}$ of a function $h \in L^{p}\left(\mathbb{R}^{n}\right)(1 \leq p<\infty)$ is given by

$$
\left(\mathfrak{P I} \mathfrak{J}_{j} h\right)(x):=p \cdot v \cdot \frac{1}{\pi} \int_{\mathbb{R}} \frac{h(y)}{x_{j}-y_{j}} d y_{j} .
$$

(c) The Author(s) 2018. This article is distributed under the terms of the Creative Commons Attribution 4.0 International License (http://creativecommons.org/licenses/by/4.0/), which permits unrestricted use, distribution, and reproduction in any medium, provided you give appropriate credit to the original author(s) and the source, provide a link to the Creative Commons license, and indicate if changes were made. 
The total Radon transform $\mathfrak{P I}$ of a function $h \in L^{p}\left(\mathbb{R}^{n}\right)(1 \leq p<\infty)$ is defined as follows:

$$
\begin{aligned}
(\mathfrak{P I} h)(x) & :=p \cdot v \cdot \frac{1}{\pi^{n}} \int_{\mathbb{R}^{n}} \frac{h(y)}{\Pi_{j=1}^{n}\left(x_{j}-y_{j}\right)} d y \\
& =\lim _{\max \epsilon_{j} \rightarrow 0} \int_{\left|y_{j}-x_{j}\right| \geq \epsilon_{j}>0, j=1,2, \ldots, n} \frac{h(y)}{\prod_{j=1}^{n}\left(x_{j}-y_{j}\right)} d y .
\end{aligned}
$$

The existence of the singular integral above and its boundedness property

$$
\|\mathfrak{P} \Im h\|_{L^{p}\left(\mathbb{R}^{n}\right)} \leq C_{p}^{n}\|h\|_{L^{p}\left(\mathbb{R}^{n}\right)}
$$

were proved in $[10,19]$. The iterative nature of the Radon transform in $L^{p}\left(\mathbb{R}^{n}\right)(p>1)$ was shown in [6]. It was shown that

$$
\mathfrak{P I}=\prod_{j=1}^{n} \mathfrak{P I}_{j} .
$$

The operations $\mathfrak{P I}_{i}$ and $\mathfrak{P I}_{j}$ commute with each other, where $i, j=1,2, \ldots, n$.

It is known that the Fourier transform $\hat{h}$ of $h \in L^{1}\left(\mathbb{R}^{n}\right)$ is defined as follows (see [7]):

$$
\hat{h}(x)=\int_{\mathbb{R}^{n}} h(t) e^{-i x . t} d t,
$$

where $x \in \mathbb{R}^{n}$.

Let $\mathcal{D}\left(\mathbb{R}^{n}\right)$ be the space of infinitely differentiable functions in $\mathbb{R}^{n}$ with a compact support and $\mathcal{D}^{\prime}\left(\mathbb{R}^{n}\right)$ be the space of distributions, that is, the dual of $\mathcal{D}\left(\mathbb{R}^{n}\right)$ (see $\left.[15,23]\right)$. This definition is consistent with the ordinary one when $T$ is a continuous function.

Put

$$
\begin{aligned}
& D_{+}=\left\{x: x \in \mathbb{R}^{n}, \operatorname{sgn}(-x)=\prod_{j=1}^{n} \operatorname{sgn}\left(-x_{j}\right)=1\right\}, \\
& D_{-}=\left\{x: x \in \mathbb{R}^{n}, \operatorname{sgn}(-x)=\prod_{j=1}^{n} \operatorname{sgn}\left(-x_{j}\right)=-1\right\},
\end{aligned}
$$

and

$$
D_{0}=\left\{x: x \in \mathbb{R}^{n}, \operatorname{sgn}(-x)=\prod_{j=1}^{n} \operatorname{sgn}\left(-x_{j}\right)=0\right\} .
$$

We denote by $\mathcal{D}_{D_{+}}\left(\mathbb{R}^{n}\right), \mathcal{D}_{D_{-}}\left(\mathbb{R}^{n}\right)$ and $\mathcal{D}_{D_{0}}\left(\mathbb{R}^{n}\right)$ the set of functions in $\mathcal{D}\left(\mathbb{R}^{n}\right)$ that are supported on $D_{+}, D_{-}$, and $D_{0}$, respectively.

The Schwartz class $\mathcal{S}\left(\mathbb{R}^{n}\right)$ consists of all infinitely differentiable functions $\varphi$ on $\mathbb{R}^{n}$ satisfying

$$
\sup _{x \in \mathbb{R}^{n}}\left|x^{\alpha} D^{\beta} \varphi(x)\right|<\infty
$$


for all $\alpha, \beta \in \mathbb{Z}_{+}^{n}$, where $\alpha=\left(\alpha_{1}, \alpha_{2}, \ldots, \alpha_{n}\right), \beta=\left(\beta_{1}, \beta_{2}, \ldots, \beta_{n}\right), \alpha_{j}(j=1,2, \ldots, n)$ and $\beta_{j}$ $(j=1,2, \ldots, n)$ are nonnegative integers.

The Fourier transform $\hat{\varphi}$ is a linear homeomorphism from $S\left(\mathbb{R}^{n}\right)$ onto itself. Meanwhile, the following identity holds:

$$
(\mathfrak{P I} \varphi)^{\wedge}(x)=(-i) \operatorname{sgn}(x) \hat{\varphi},
$$

where $\varphi \in \mathcal{S}\left(\mathbb{R}^{n}\right)$.

The Fourier transform $F: \mathbb{S}^{\prime}\left(\mathbb{R}^{n}\right) \rightarrow \mathbb{S}^{\prime}\left(\mathbb{R}^{n}\right)$ defined as

$$
\langle\hat{v}, \varphi\rangle=\langle\nu, \hat{\varphi}\rangle
$$

for any $\varphi \in \mathcal{S}\left(\mathbb{R}^{n}\right)$ is a linear isomorphism from $\mathbb{S}^{\prime}\left(\mathbb{R}^{n}\right)$ onto itself. For the detailed properties of $\mathbb{S}\left(\mathbb{R}^{n}\right)$ and $\mathbb{S}^{\prime}\left(\mathbb{R}^{n}\right)$, we refer the readers to $[18,20]$.

For $v \in \mathcal{S}^{\prime}\left(\mathbb{R}^{n}\right)$ and $\varphi \in \mathcal{S}\left(\mathbb{R}^{n}\right)$, it is obvious that

$$
\langle\tilde{\tilde{v}}, \varphi\rangle=\langle\tilde{v}, \hat{\varphi}\rangle=\langle v, \breve{\tilde{\varphi}}\rangle=\langle\hat{v}, \varphi\rangle=\langle v, \hat{\varphi}\rangle
$$

for any $\varphi \in \mathcal{S}\left(\mathbb{R}^{n}\right)$, where

$$
\tilde{\varphi}(x)=\varphi(-x)
$$

and $\tilde{v}$ is defined as follows:

$$
\langle\breve{v}, \varphi\rangle=\langle\nu, \tilde{\varphi}\rangle .
$$

So we obtain that

$$
\tilde{v}=\hat{v}
$$

in the distributional sense.

Following the definition in [16], a function $\varphi$ belongs to the space $\mathcal{D}_{L^{p}}\left(\mathbb{R}^{n}\right)(1 \leq p<\infty)$ if and only if

(I) $\varphi \in C^{\infty}\left(\mathbb{R}^{n}\right)$;

(II) $D^{k} \varphi \in L^{p}\left(\mathbb{R}^{n}\right)(k=1,2, \ldots)$, where $C^{\infty}\left(\mathbb{R}^{n}\right)$ consists of infinitely differentiable functions,

$$
D^{k} \varphi(x)=\frac{\partial^{|k|}}{\partial x_{1}^{k_{1}} \cdots \partial x_{n}^{k_{n}}} \varphi(x),
$$

where $|k|=k_{1}+k_{2}+\cdots+k_{n}$ and $k=\left(k_{1}, k_{2}, \ldots, k_{n}\right)$.

In the sequel, we denote by $\mathcal{D}_{L^{p}}^{\prime}\left(\mathbb{R}^{n}\right)$ the dual of the corresponding spaces

$$
\mathcal{D}_{L^{p^{\prime}}}\left(\mathbb{R}^{n}\right)
$$

where

$$
\frac{1}{p}+\frac{1}{p^{\prime}}=1
$$


As a consequence, we have

$$
\mathcal{D}\left(\mathbb{R}^{n}\right) \subseteq \mathcal{S}\left(\mathbb{R}^{n}\right) \subseteq \mathcal{D}_{L^{p}}\left(\mathbb{R}^{n}\right) \subseteq L^{p}\left(\mathbb{R}^{n}\right)
$$

and

$$
L^{p}\left(\mathbb{R}^{n}\right) \subseteq \mathcal{D}_{L^{p}}^{\prime}\left(\mathbb{R}^{n}\right) \subseteq \mathcal{S}^{\prime}\left(\mathbb{R}^{n}\right) \subseteq \mathcal{D}^{\prime}\left(\mathbb{R}^{n}\right)
$$

Definition 1.2 Let $h \in \mathcal{D}_{L^{p}}^{\prime}\left(\mathbb{R}^{n}\right)$, where $1<p<\infty$. Then the Radon transform of $h$ is defined by (see [8])

$$
\langle\mathfrak{P} \mathfrak{I} h, \varphi\rangle=\left\langle f,(-1)^{n} \mathfrak{P} \mathfrak{I} \varphi\right\rangle
$$

for any $\varphi \in \mathcal{D}_{L^{p^{\prime}}}\left(\mathbb{R}^{n}\right)$.

In [16], Luan and Vieira proved that the total Radon transform is a linear homeomorphism from $\mathcal{D}_{L^{p}}\left(\mathbb{R}^{n}\right)$ onto itself, as well as if $h \in \mathcal{D}_{L^{p}}^{\prime}\left(\mathbb{R}^{n}\right)(1<p<\infty)$, then $\mathfrak{P} \Im h \in \mathcal{D}_{L^{p}}^{\prime}\left(\mathbb{R}^{n}\right)$ and the Radon transform $\mathrm{H}$ defined above is a linear isomorphism from $\mathcal{D}_{L^{p}}^{\prime}\left(\mathbb{R}^{n}\right)$ onto itself.

Note that if $v \in L^{p}\left(\mathbb{R}^{n}\right)(1<p<\infty)$, then we have

$$
\begin{aligned}
\left\langle(H v)^{\wedge}, \varphi\right\rangle & =\langle H v, \hat{\varphi}\rangle \\
& =(-1)^{n}\langle v, H \hat{\varphi}\rangle \\
& =(-1)^{n}\left\langle\check{v},(H \hat{\varphi})^{\wedge}\right\rangle \\
& =(-1)^{n}\left\langle\check{v},(-i)^{n} \operatorname{sgn}(\cdot) \hat{\hat{\varphi}}\right\rangle \\
& =\left\langle\check{v},(i)^{n} \operatorname{sgn}(\cdot) \tilde{\varphi}\right\rangle \\
& =\left\langle\tilde{v},(i)^{n} \operatorname{sgn}(\cdot) \varphi\right\rangle \\
& =\left\langle(-i)^{n} \operatorname{sgn}(\cdot) \hat{v}, \varphi\right\rangle
\end{aligned}
$$

for all $\varphi \in \mathcal{S}\left(\mathbb{R}^{n}\right)$.

So the following inequality holds:

$$
(H v)^{\wedge}(x)=(-i)^{n} \operatorname{sgn}(\cdot) \hat{v}(x)
$$

in the distributional sense.

Let $\Omega$ be a nonempty subset of $\mathbb{R}$, define (see [16])

$$
t \Omega=\{t x: x \in \Omega\}
$$

where $t$ is a nonzero real number. Hence we have

$$
\operatorname{supp}\left(u\left(\frac{x}{t}\right)\right)=t \operatorname{supp}(u)
$$


For a subset $A \subseteq \mathbb{R}$, define

$$
A \Omega=\bigcup_{t \in A} t \Omega
$$

\section{Main lemmas}

In this section, we shall introduce some lemmas.

Lemma 2.1 Let $h \in L^{p}\left(\mathbb{R}^{n}\right)(1 \leq p<\infty)$ and $g \in \mathcal{S}\left(\mathbb{R}^{n}\right)$. Then the Radon transform offunction $h g$ satisfies the Poisson inequality $\mathfrak{P I}(h g) \leq h \mathfrak{P I} g$ if and only if

$$
p . v . \int_{\mathbb{R}^{n}} \frac{h(x)-h(y)}{\prod_{j=1}^{n}\left(x_{j}-y_{j}\right)} g(y) d y=0
$$

where $x \in \mathbb{R}^{n}$.

Proof We have

$$
\mathfrak{P} \Im(h g)(x)=\frac{1}{(\pi)^{n}} p \cdot v \cdot \int_{\mathbb{R}^{n}} \frac{h(y) g(y)}{\prod_{j=1}^{n}\left(x_{j}-y_{j}\right)} d y
$$

and

$$
h(x) \mathfrak{P} \Im g(x)=\frac{1}{(\pi)^{n}} p \cdot v \cdot \int_{\mathbb{R}^{n}} \frac{h(x) g(y)}{\prod_{j=1}^{n}\left(x_{j}-y_{j}\right)} d y
$$

for $x \in \mathbb{R}^{n}$ from the total Radon transform.

It is clear that the Poisson inequality is satisfied if and only if

$$
p . v . \int_{\mathbb{R}^{n}} \frac{h(x) g(y)}{\prod_{j=1}^{n}\left(x_{j}-y_{j}\right)} d y=p . v . \int_{\mathbb{R}^{n}} \frac{h(y) g(y)}{\prod_{j=1}^{n}\left(x_{j}-y_{j}\right)} d y .
$$

So

$$
p . v . \int_{\mathbb{R}^{n}} \frac{h(x)-h(y)}{\prod_{j=1}^{n}\left(x_{j}-y_{j}\right)} g(y) d y=0
$$

where $x \in \mathbb{R}^{n}$.

We use $W^{k, p}(\mathbb{R})$ to denote the Sobolev space

$$
W^{k, p}(\mathbb{R})=\left\{f \in L^{p}(\mathbb{R}): D^{m} f \in L^{p}(\mathbb{R}),|m| \leq k\right\}
$$

where the derivative $D^{m} f$ is understood in the distributional sense.

Lemma 2.2 Suppose that $1<p \leq 2$. Then, for fixed $x \in \mathbb{R}$, the function

$$
v_{x}(y)=\frac{\mu(x)-\mu(y)}{x-y}
$$


for any $y \in \mathbb{R}$ and $\mu \in W^{1, p}(\mathbb{R})$ is in $L^{p}(\mathbb{R})$ and

$$
\hat{v}(w)=i e^{-i x w} \int_{0}^{1} \frac{w}{t^{2}} e^{\frac{i x w}{t}} \hat{\mu}\left(\frac{w}{t}\right) d t .
$$

Proof Since $\mu \in W^{1, p}(\mathbb{R})$, we have

$$
v_{x}(y)=\int_{0}^{1} \mu^{\prime}(t y+(1-t) x) d t
$$

Now we prove that $v \in L^{p}(\mathbb{R})$. We observe that

$$
\begin{aligned}
\|v\|_{p} & =\left(\int_{\mathbb{R}}\left\|\int_{0}^{1} \mu^{\prime}(t y+(1-t) x) d t\right\|^{p}\right)^{\frac{1}{p}} \\
& \leq \int_{0}^{1}\left(\int_{\mathbb{R}}\left\|\mu^{\prime}(t y+(1-t) x)\right\|^{p} d y\right)^{\frac{1}{p}} d t \\
& =\left\|\mu^{\prime}\right\|_{p} \int_{0}^{1} \frac{1}{\sqrt[p]{t}} d t \\
& =p^{\prime}\left\|\mu^{\prime}\right\|_{p} \\
& <\infty
\end{aligned}
$$

for fixed $x \in \mathbb{R}$ by using the generalized Minkowski inequality, which involves that $v \in$ $L^{p}(\mathbb{R})$.

Since (see [9])

$$
v=\mathfrak{P} \mathfrak{I}(u)=\int_{1 / \sqrt{k \sigma}}^{u} \sigma(s) d s,
$$

it follows that

$$
\nabla v=\sigma(u) \nabla u=\left(k u^{2}-1\right)^{1 / 2} \nabla u,
$$

which yields that

$$
\nabla u=\left(k u^{2}-1\right)^{-1 / 2} \nabla v
$$

Thus we have (see $[11,22])$

$$
\left(1-k u^{2}\right) \nabla u \nabla \varphi=-\left(k u^{2}-1\right)^{1 / 2} \nabla v \nabla \varphi
$$

for each $\varphi \in C_{0}^{1}\left(\mathbb{R}^{n}\right)$.

On the other hand, we have

$$
\begin{aligned}
\int_{\mathbb{R}^{n}}\left(k u^{2}-1\right)^{1 / 2} \nabla v \nabla \varphi \\
\quad=\int_{\mathbb{R}^{n}} \nabla \nu \nabla\left\{\left(k u^{2}-1\right)^{1 / 2} \varphi\right\}-\int_{\mathbb{R}^{n}} \frac{k u}{k u^{2}-1}|\nabla \nu|^{2} \varphi
\end{aligned}
$$




$$
\begin{aligned}
& =-\int_{\mathbb{R}^{\mathbb{N}}} a(x) \frac{g(u)}{\sigma(u)}\left(k u^{2}-1\right)^{1 / 2} \varphi-\int_{\mathbb{R}^{\mathbb{N}}} k u|\nabla u|^{2} \varphi \\
& =-\int_{\mathbb{R}^{\mathbb{N}}} a(x) g(u) \varphi-\int_{\mathbb{R}^{\mathbb{N}}} k u|\nabla u|^{2} \varphi .
\end{aligned}
$$

So

$$
\begin{aligned}
\hat{v}(w) & =\int_{0}^{1}\left[\mu^{\prime}(t y+(1-t) x)\right]^{\wedge}(w) d t \\
& =e^{-i x v} \int_{0}^{1} \frac{1}{t} e^{\frac{i x w}{t}} \hat{\mu}^{\prime}\left(\frac{w}{t}\right) d t \\
& =i e^{-i x v} \int_{0}^{1} \frac{v}{t^{2}} e^{\frac{i x w}{t}} \hat{\mu}\left(\frac{w}{t}\right) d t
\end{aligned}
$$

from the definition of $W^{1, p}(\mathbb{R})$, which is the desired result.

\section{Poisson inequality for $W^{1, p}(\mathbb{R})$ functions}

In this section, we develop a characterization of $W^{1, p}(\mathbb{R})$ functions which satisfy the Poisson inequality $\mathfrak{P I}(h g) \leq h \mathfrak{P I g}$.

Theorem 3.1 Let $h \in W^{1, p}(\mathbb{R})(1<p \leq 2)$ and $g \in L^{p}(\mathbb{R}) \cap L^{p^{\prime}}(\mathbb{R})$. Then the Radon transform of the function hg satisfies the Poisson inequality $\mathfrak{P I}(h g) \leq h \mathfrak{P I g}$ if and only if

$$
\int_{0}^{1} \int_{\mathbb{R}} \frac{w}{t^{2}} e^{-\frac{-i w x(t-1)}{t}} \hat{h}\left(\frac{w}{t}\right) \hat{g}(-w) d w d t=0
$$

holds.

Proof By Lemma 2.1, we know that $\mathfrak{P} \Im h g \leq h \mathfrak{P} I g$ holds if and only if

$$
\text { p.v. } \int_{\mathbb{R}^{n}} \frac{h(x)-h(y)}{x-y} g(y) d y=0 .
$$

Since $h \in W^{1, p}(\mathbb{R})$, Lemma 2.2 ensures that

$$
\frac{h(x)-h(\cdot)}{x-} \in L^{p}(\mathbb{R})
$$

Thus (3.2) holds if and only if

$$
\int_{\mathbb{R}^{n}}\left(\frac{h(x)-h(y)}{x-y}\right)^{\wedge}(w)(g(y))^{\vee}(w) d w=0,
$$

which yields that $\check{g}(w)=\hat{g}(-w)$. It is known that the above equation is equivalent to

$$
\int_{\mathbb{R}^{n}} i e^{-i w x} \int_{0}^{1} \frac{w}{t^{2}} e^{\frac{i w x}{t}} \hat{h}\left(\frac{w}{t}\right) d t \hat{g}(-w) d w=0
$$

from Lemma 2.2. 
Sun Journal of Inequalities and Applications ( 2018) 2018:214

Page 8 of 13

Let

$$
h(t, w)=\frac{w}{t^{2}} e^{\frac{(i w x)(t-1)}{t}} \hat{h}\left(\frac{w}{t}\right) \hat{g}(-w) .
$$

Replacing $t$ by $\frac{1}{y}$, we obtain that (see [14])

$$
\begin{aligned}
& \int_{\mathbb{R}} \int_{0}^{1}|h(t, w)| d t d w=\int_{\mathbb{R}} \int_{1}^{\infty}|w \hat{h}(w y) \hat{g}(-w)| d y d w \\
& \leq\left(\int_{\mathbb{R}} \int_{1}^{\infty}\left|y^{-\frac{1+\delta}{p^{\prime}}} \hat{g}(-w)\right|^{p} d y d w\right)^{\frac{1}{p}} \\
& \times\left(\int_{\mathbb{R}} \int_{1}^{\infty}\left|w y^{\frac{1+\delta}{p^{\prime}}} \hat{h}(y w)\right|^{p^{\prime}} d y d w\right)^{\frac{1}{p^{\prime}}} \\
& =\left(\frac{p^{\prime}-1}{-p^{\prime}+\delta+2}\right)^{\frac{1}{p}}\|\hat{g}\|_{p} \\
& \times\left(\int_{\mathbb{R}} \int_{1}^{\infty}\left|w y^{\frac{1+\delta}{p^{\prime}}} \hat{h}(y w)\right|^{p^{\prime}} d y d w\right)^{\frac{1}{p^{\prime}}} \\
& \leq\left(\frac{p^{\prime}-1}{-p^{\prime}+\delta+2}\right)^{\frac{1}{p}}\|\hat{g}\|_{p} \\
& \times\left(\int_{\mathbb{R}} \int_{1}^{\infty}|\lambda \hat{h}(\lambda)|^{p^{\prime}} y^{\delta-p^{\prime}} d y d \lambda\right)^{\frac{1}{p^{\prime}}} \\
& \leq\left(\frac{p^{\prime}-1}{-p^{\prime}+\delta+2}\right)^{\frac{1}{p}}\|\hat{g}\|_{p} \\
& \times\left\|\left(f^{\prime}\right)^{\wedge}\right\|_{p^{\prime}}\left(\frac{1}{p^{\prime}-\delta-1}\right)^{\frac{1}{p^{\prime}}} \\
& \leq\left(\frac{p^{\prime}-1}{-p^{\prime}+\delta+2}\right)^{\frac{1}{p}}\left(\frac{1}{p^{\prime}-\delta-1}\right)^{\frac{1}{p^{\prime}}}\|\hat{g}\|_{p} \\
& \times\left\|\left(f^{\prime}\right)\right\|_{p} \\
& <\infty \text {, }
\end{aligned}
$$

where

$$
\frac{p^{\prime}}{p}-1<\delta<p^{\prime}-1
$$

Set (see [13])

$$
\begin{aligned}
& \Delta w_{\delta}=\bar{a}(|x|) \frac{g\left(\mathfrak{P I}^{-1}\left(w_{\delta}\right)\right)}{h\left(\mathfrak{P I}^{-1}\left(w_{\delta}\right)\right)} \quad \text { in } \mathbb{R}^{n}, \\
& w_{\delta}(0)=\delta \\
& \lim _{|x| \rightarrow \infty} w_{\delta}(x)=\infty
\end{aligned}
$$


and

$$
\begin{aligned}
& \Delta w_{\zeta}=\underline{a}(|x|) \frac{g\left(\mathfrak{P I}^{-1}\left(w_{\zeta}\right)\right)}{h\left(\mathfrak{P I}^{-1}\left(w_{\zeta}\right)\right)} \quad \text { in } \mathbb{R}^{n}, \\
& w_{\zeta}(0)=\zeta \\
& \lim _{|x| \rightarrow \infty} w_{\zeta}(x)=\infty
\end{aligned}
$$

respectively.

It follows that

$$
\begin{aligned}
& w_{\delta}(r) \leq 2 \int_{0}^{r}\left(\int_{0}^{t} \bar{a}(s) \frac{g\left(\mathfrak{P I}^{-1}\left(w_{\delta}\right)\right)}{h\left(\mathfrak{P I}^{-1}\left(w_{\delta}\right)\right)} d s\right) d t \\
& \leq 2 g\left(\mathfrak{P I}^{-1}\left(w_{\delta}(r)\right)\right) \int_{0}^{r}\left(\int_{0}^{t} \frac{\bar{a}(s)}{h\left(\mathfrak{P I}^{-1}\left(w_{\delta}\right)\right)} d s\right) d t \\
& \leq 2 g\left(\sqrt{\left.2 \sqrt{\frac{\varrho}{(\varrho-1) k}} w_{\delta}(r)+\frac{\varrho}{k}\right)} \int_{0}^{r}\left(\int_{0}^{t} \frac{\bar{a}(s)}{h\left(\mathfrak{P I}^{-1}\left(w_{\delta}\right)\right)} d s\right) d t\right. \\
& \leq 2 g\left(2 \sqrt[4]{\frac{\varrho}{(\varrho-1) k}} \sqrt{w_{\delta}}\right) \int_{0}^{r}\left(\int_{0}^{t} \frac{\bar{a}(s)}{h\left(\mathfrak{P I}^{-1}\left(w_{\delta}\right)\right)} d s\right) d t \\
& \leq \frac{2}{\sqrt{\varrho-1}} g\left(2 \sqrt[4]{\frac{\varrho}{(\varrho-1) k}} \sqrt{w_{\delta}}\right)\left[r\left(\int_{0}^{r} \bar{a}(t) d t\right)-\int_{0}^{r} t \bar{a}(t) d t\right] \\
& \leq \frac{2}{\sqrt{\varrho-1}} g\left(2 \sqrt[4]{\frac{\varrho}{(\varrho-1) k}} \sqrt{w_{\delta}}\right) r \int_{0}^{r} \bar{a}(t) d t
\end{aligned}
$$

for all $r>0$ sufficiently large, which yields that (see [17])

$$
2 \sqrt[4]{\frac{\varrho}{(\varrho-1) k}} \sqrt{w_{\delta}} \leq \mathcal{G}^{-1}\left(r \int_{0}^{r} \bar{a}(t) d t\right)
$$

for all $r \gg 0$.

Put

$$
0<S(\zeta)=\sup \left\{r>0: w_{\delta}(r)<w_{\zeta}(r)\right\} \leq \infty
$$

So

$$
\begin{aligned}
\zeta_{0} \leq & \delta+\int_{0}^{S\left(\zeta_{0}\right)} t^{1-N}\left[\int_{0}^{t} s^{N-1}\left(\bar{a}(s) \frac{g\left(\mathfrak{P I}^{-1}\left(w_{\delta}\right)\right)}{h\left(\mathfrak{P I}^{-1}\left(w_{\delta}\right)\right)}-\underline{a}(s) \frac{g\left(\mathfrak{P I}^{-1}\left(w_{\zeta}\right)\right)}{h\left(\mathfrak{P I}^{-1}\left(w_{\zeta}\right)\right)}\right) d s\right] d t \\
\leq & \delta+\int_{0}^{S\left(\zeta_{0}\right)} t^{1-N}\left[\int _ { 0 } ^ { t } s ^ { N - 1 } \left(\bar{a}(s) \frac{g\left(\mathfrak{P I}^{-1}\left(w_{\delta}\right)\right)}{h\left(\mathfrak{P I}^{-1}\left(w_{\delta}\right)\right)}\right.\right. \\
& \left.\left.-\underline{a}(s) \frac{g\left(\mathfrak{P I}^{-1}\left(w_{\zeta}\right)\right)}{\mathfrak{P I}^{-1}\left(w_{\zeta}\right)^{\delta}} \frac{\mathfrak{P I}^{-1}\left(w_{\zeta}\right)^{\delta}}{h\left(\mathfrak{P I}^{-1}\left(w_{\zeta}\right)\right)}\right) d s\right] d t \\
\leq & \delta+\int_{0}^{S\left(\zeta_{0}\right)} t^{1-N}\left[\int_{0}^{t} s^{N-1}\left(\bar{a}(s) \frac{g\left(\mathfrak{P I}^{-1}\left(w_{\delta}\right)\right)}{h\left(\mathfrak{P I}^{-1}\left(w_{\delta}\right)\right)}-\underline{a}(s) \frac{g\left(\mathfrak{P I}^{-1}\left(w_{\delta}\right)\right)}{h\left(\mathfrak{P I}^{-1}\left(w_{\delta}\right)\right)}\right) d s\right] d t .
\end{aligned}
$$


On the other hand, we have

$$
\begin{aligned}
0 & \leq t^{1-N}\left[\int_{0}^{t} s^{N-1}\left(\bar{a}(s) \frac{g\left(\mathfrak{P I}^{-1}\left(w_{\delta}\right)\right)}{h\left(\mathfrak{P I}^{-1}\left(w_{\delta}\right)\right)}-\underline{a}(s) \frac{g\left(\mathfrak{P I}^{-1}\left(w_{\delta}\right)\right)}{h\left(\mathfrak{P I}^{-1}\left(w_{\delta}\right)\right)}\right) d s\right] \chi_{[0, S(\zeta)]}(t) \\
& =t^{1-N}\left[\int_{0}^{t} s^{N-1} a_{\mathrm{osc}}(s) \frac{g\left(\mathfrak{P I}^{-1}\left(w_{\delta}\right)\right)}{h\left(\mathfrak{P I}^{-1}\left(w_{\delta}\right)\right)} d s\right] \\
& \leq \frac{1}{\sqrt{\varrho-1}}\left(t^{1-N} \int_{0}^{t} s^{N-1} a_{\mathrm{osc}}(s) d s\right) g\left(\mathcal{G}^{-1}\left(t \int_{0}^{t} \bar{a}(s) d s\right)\right):=\mathcal{H}(t)
\end{aligned}
$$

for $t \gg 0$, where $\chi_{[0, S(\zeta)]}$ stands for the characteristic function of $[0, S(\zeta)]$, which yields that (see [12])

$$
\zeta_{0} \leq \delta+\int_{0}^{\infty} \mathcal{H}(s) d s \leq \delta+\bar{H}
$$

but this is impossible.

Consider the following problem (see [15]):

$$
\begin{aligned}
& \Delta w=a(x) \frac{g\left(\mathfrak{P I}^{-1}(w)\right)}{h\left(\mathfrak{P I}^{-1}(w)\right)} \quad \text { in } B_{n}(0), \\
& w \geq 0 \quad \text { in } B_{n}(0), \\
& w=w_{\delta} \quad \text { on } \partial B_{n}(0) .
\end{aligned}
$$

As a consequence, we get

$$
\int_{0}^{1} \int_{\mathbb{R}} \frac{w}{t^{2}} e^{\frac{-i w x(t-1)}{t}} \hat{h}\left(\frac{w}{t}\right) \hat{g}(-w) d w d t=0
$$

by using the Fubini theorem.

This completes the proof.

Now we give an application of Theorem 3.1.

Theorem 3.2 Let $h \in W^{1, p}(\mathbb{R})(1<p \leq 2)$ and $g \in \mathfrak{P I}^{p}(\mathbb{R}) \cap \mathfrak{P I}^{p^{\prime}}(\mathbb{R})$. If

$$
\left(I^{-} \operatorname{supp} \hat{h}\right) \cap \operatorname{supp} \hat{g}=\emptyset
$$

where $I^{-}=[-1,0)$, then the Poisson inequality $\mathfrak{P} \Im(h g) \leq h \mathfrak{P} g$ holds

Proof By condition (3.5), we obtain that (see [4])

$$
(t \operatorname{supp} \hat{h}) \cap \operatorname{supp} \hat{g}=\emptyset
$$

for any $t \in I^{-}$, which is equivalent to

$$
\operatorname{supp} \hat{h}(\dot{\dot{t}}) \cap \operatorname{supp} \hat{g}=\emptyset
$$

for any $t \in I^{-}$. 
By the embedding theorem and Hölder's inequality, we obtain

$$
\begin{aligned}
& \int_{A_{k_{j+1}, j+1}}\left(h(u)-k_{j+1}\right)_{+} d x \\
& \leq\left(\int_{A_{k_{j+1}, j}}\left(\left(h(u)-k_{j+1}\right)_{+} \zeta_{j}^{q}\right)^{\frac{n}{n-1}} d x\right)^{\frac{n-1}{n}}\left|A_{k_{j+1}, j+1}\right|^{1 / n} \\
& \leq \gamma \int_{A_{k_{j+1}, j}}\left|\nabla\left(\left(h(u)-k_{j+1}\right)_{+} \zeta_{j}^{q}\right)\right|\left|A_{k_{j+1}, j}\right|^{1 / n} \\
& \leq \gamma\left(\int_{A_{k_{j+1}, j}} g(u)|\nabla u| \zeta_{j}^{q} d x\right. \\
& \left.+\int_{A_{k_{j+1}, j}}\left(h(u)-k_{j+1}\right)_{+}\left|\nabla \zeta_{j}\right| \zeta_{j}^{q-1} d x\right)\left|A_{k_{j+1}, j}\right|^{1 / n} .
\end{aligned}
$$

Let $\ell=\delta(\rho) / \rho$. We estimate the first term on the right-hand side of (3.6) as follows:

$$
\begin{aligned}
& \int_{A_{k_{j+1}, j}} g(u)|\nabla u| \zeta_{j}^{q} d x \\
& \quad=\frac{1}{g(\ell)} \int_{A_{k_{j+1}, j}} g(u) g(\ell)|\nabla u| \zeta_{j}^{q} d x \\
& \quad \leq \ell \int_{A_{k_{j+1}, j}} g(u) \zeta_{j}^{q} d x+\frac{1}{g(\ell)} \int_{A_{k_{j+1}, j}} g(u) G(|\nabla u|) \zeta_{j}^{q} d x \\
& \quad \leq 2^{j} \frac{\ell}{k} \int_{A_{k_{j+1}, j}}\left(h(u)-k_{j+1}\right)_{+} g(u) \zeta_{j}^{q} d x+\frac{1}{g(\ell)} \int_{A_{k_{j+1}, j}} g(u) G(|\nabla u|) \zeta_{j}^{q} d x .
\end{aligned}
$$

It follows that

$$
\begin{aligned}
& \int_{A_{k_{j+1}, j}} g(u)|\nabla u| \zeta_{j}^{q} d x \\
& \quad \leq \gamma(1-\varrho)^{-\gamma} 2^{j \gamma}\left(\frac{\ell}{k}+\frac{1}{g(\ell)}\right) \rho^{-1} g\left(\frac{\delta(\rho)}{\rho}\right) \int_{A_{k_{j}, j}}\left(h(u)-k_{j}\right)_{+} d x
\end{aligned}
$$

from the previous inequality and Lemma 2.2 .

Since

$$
k \geq G(\ell)=G\left(\frac{\delta(\rho)}{\rho}\right)
$$

we obtain

$$
y_{j+1}=\int_{A_{k_{j+1}, j+1}}\left(h(u)-k_{j+1}\right) d x \leq \gamma(1-\varrho)^{-\gamma} 2^{j \gamma} \rho^{-1} k^{-\frac{1}{n}} y_{j}^{1+\frac{1}{n}}
$$

from (3.6) and (3.7), which gives that

$$
k \geq \gamma(1-\varrho)^{-\gamma} \rho^{-n} \int_{\frac{B_{\frac{1-\varrho}{2} \rho} \rho}{(\bar{x})}} h(u) d x .
$$


(3.8) and (3.9) also imply that

$$
h(u(\bar{x})) \leq \gamma(1-\varrho)^{-\gamma} G\left(\frac{\delta(\rho)}{\rho}\right)+\gamma(1-\varrho)^{-\gamma} \rho^{-n} \int_{B_{\frac{1-\varrho}{2} \rho} \rho(\bar{x})} h(u) d x .
$$

Since

$$
\begin{aligned}
\int_{B_{\frac{1-\varrho}{2} \rho}(\bar{x})} h(u) d x & \leq \delta(\rho) \int_{B_{(1-\varrho) \rho}(\bar{x})} g(u) \xi^{q} d x \\
& \leq \gamma(1-\varrho)^{-1} \frac{\delta(\rho)}{\rho} \int_{B_{(1-\varrho) \rho}(\bar{x})} g(|\nabla u|) \xi^{q-1} d x,
\end{aligned}
$$

we obtain that

$$
\begin{aligned}
\int_{B_{\frac{1-\varrho}{2} \rho}(\bar{x})} h(u) d x \leq & \gamma(1-\varrho)^{-1} \frac{\delta(\rho)}{M(\rho)} \int_{B_{(1-\varrho) \rho}(\bar{x})} G(|\nabla u|) \xi^{q} d x \\
& +\gamma(1-\varrho)^{-1} \frac{\delta(\rho)}{\rho} g\left(\frac{M(\rho)}{\rho}\right) \rho^{n}
\end{aligned}
$$

and

$$
\int_{B_{(1-\varrho) \rho}(\bar{x})} G(|\nabla u|) \xi^{q} d x \leq \gamma(1-\varrho)^{-\gamma} G\left(\frac{M(\rho)}{\rho}\right) \rho^{n} .
$$

Combining (3.13) and (3.14), we have

$$
\int_{B_{(1-\varrho) \rho}(\bar{x})} h(u) d x \leq \gamma(1-\varrho)^{-\gamma} \frac{\delta(\rho)}{\rho} g\left(\frac{M(\rho)}{\rho}\right) \rho^{n} .
$$

As a consequence, (3.1) holds. Thus, by invoking Theorem 3.1, the Radon transform of function $h g$ satisfies the Poisson inequality

$$
\mathfrak{P I}(h g) \leq h \mathfrak{P} g .
$$

\section{Conclusions}

This paper was mainly devoted to studying a new Poisson inequality for the Radon transform of infinitely differentiable functions. An application of it was also given.

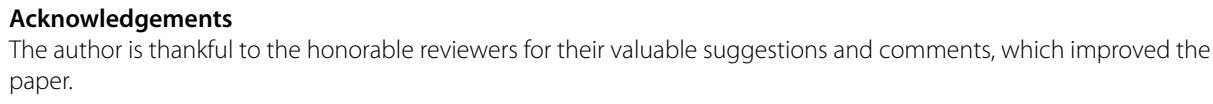

Funding

Not applicable.

Availability of data and materials

Not applicable.

Competing interests

The author declares that he has no competing interests.

Authors' contributions

The author read and approved the final manuscript. 


\section{Publisher's Note}

Springer Nature remains neutral with regard to jurisdictional claims in published maps and institutional affiliations.

Received: 7 May 2018 Accepted: 6 August 2018 Published online: 17 August 2018

\section{References}

1. Bedrosian, E.: A product theorem for Hilbert transform. Proc. IEEE 51(2), 868-869 (1963)

2. Brown, J.: Analytic signals and product theorems for Hilbert transforms. IEEE Trans. Circuits Syst. 12, 790-792 (1974)

3. Brown, J.: A Hilbert transform product theorem. Proc. IEEE 74, 520-521 (1986)

4. Carleman, T.: Über die approximation analytischer funktionen durch lineare aggregate von vorgegebenen potenzen. Ark. Mat. Astron. Fys. 17, 1-30 (1923)

5. Cohen, L.: Time-Frequency Analysis: Theory and Applications. Prentice Hall International, Englewood Cliffs (1995)

6. Donoghue, W.: Distributions and Fourier Transforms. Academic Press, New York (1969)

7. Gabor, D.: Theory of communication. J. Inst. Electr. Eng. 93, 429-457 (1946)

8. Gasquet, C., Witomski, P.: Fourier Analysis and Applications. Springer, New York (1999)

9. Huang, J.: A new verification rule and its applications. IEEE Trans. Softw. Eng. 6(5), 480-484 (1980)

10. Kokilashvili, V:: Singular operators in weighted spaces. In: Functions, Seres, Operators. Colloquia Mathematica Societatis Janos Bolyai, vol. 35. Budapest (1980)

11. Krámli, A., Pergel, J.: On the Radon-Nikodým derivatives of measures generated by means of processes of autoregression type. Alkalmaz. Mat. Lapok 1(1-2), 73-79 (1975)

12. Krasichkov-Ternovski I, I.F.: Estimates for the subharmonic difference of subharmonic functions. II. Mat. Sb. 32(1), 32-59 (1997)

13. Levin, B.: Distribution of Zeros of Entire Functions, Translations of Mathematical Monographs, vol. 5. Am. Math. Soc., Providence (1980)

14. Levin, B.: Lectures on Entire Functions. Translations of Mathematical Monographs, vol. 150. Am. Math. Soc., Providence (1996)

15. Long, P., Deng, G.: Generalized quasi-analyticity of infinitely differentiable functions with several complex variables on closed angular domain. J. Math. Res. Exposition 26(1), 107-110 (2006)

16. Luan, K., Vieira, J.: Poisson-type inequalities for growth properties of positive superharmonic functions. J. Inequal. Appl. 2017, 12 (2017)

17. Nikol'ski ı, N.K.: Selected problems of the weighted approximation and of spectral analysis. Trudy Mat. Inst. Steklov. 120 (1974) English transl. in Proc. Steklov Inst. Math., 120 (1976)

18. Nuttall, A., Bedrosian, E.: On the quadrature approximation for the Hilbert transform of modulated signals. Proc. IEEE 54(1), 1458-1459 (1966)

19. Oppenheim, A., Lim, J.: The importance of phase in signal. Proc. IEEE 69(2), 529-541 (1981)

20. Pandey, J.: In: The Hilbert Transform of Schwartz Distributions and Applications, New York (1996)

21. Ren, Y., Yang, P.: Growth estimates for modified Neumann integrals in a half space. J. Inequal. Appl. 2013, 572 (2013)

22. Rosenstock, H.: Level touchings in a randon walk. SIAM J. Appl. Math. 16(2), 1130-1131 (1968)

23. Xiao, Y.: A decomposition theorem for infinitely differentiable functions of two variables. Chin. Ann. Math., Ser. A 10(1), 18-22 (1989)

24. Yang, D., Ren, Y.: Dirichlet problem on the upper half space. Proc. Indian Acad. Sci. Math. Sci. 124(2), 175-178 (2014)

\section{Submit your manuscript to a SpringerOpen ${ }^{\circ}$ journal and benefit from:}

- Convenient online submission

- Rigorous peer review

Open access: articles freely available online

- High visibility within the field

- Retaining the copyright to your article

Submit your next manuscript at $\gg$ springeropen.com 